\title{
Endosphenoidal coil for intraoperative magnetic resonance imaging of the pituitary gland during transsphenoidal surgery
}

\author{
Prashant Chittiboina, MD, MPH, ${ }^{1}$ S. Lalith Talagala, PhD, ${ }^{2}$ Hellmut Merkle, $\mathrm{PhD},{ }^{3}$ \\ Joelle E. Sarlls, PhD, ${ }^{2}$ Blake K. Montgomery, BA, ${ }^{1}$ Martin G. Piazza, MS, ${ }^{1}$ \\ Gretchen Scott, BSN, RN, ${ }^{1}$ Abhik Ray-Chaudhury, MD, ${ }^{1}$ Russell R. Lonser, MD, ${ }^{1,5}$ \\ Edward H. Oldfield, MD, ${ }^{1,6}$ Alan P. Koretsky, PhD, ${ }^{3}$ and John A. Butman, MD, PhD ${ }^{4}$
}

${ }^{1}$ Surgical Neurology Branch, National Institute of Neurological Disorders and Stroke; ${ }^{2} \mathrm{NIH}$ MRI Research Facility; ${ }^{3}$ Laboratory of Functional and Molecular Imaging, National Institute of Neurological Disorders and Stroke; and ${ }^{4}$ Radiology and Imaging Sciences, the National Institutes of Health, Bethesda, Maryland; ${ }^{5}$ Department of Neurological Surgery, The Ohio State University Wexner Medical Center, Columbus, Ohio; and ${ }^{6}$ Department of Neurological Surgery, University of Virginia Health System, Charlottesville, Virginia

OBJECTIVE Pituitary MR imaging fails to detect over $50 \%$ of microadenomas in Cushing's disease and nearly $80 \%$ of cases of dural microinvasion. Surface coils can generate exceptionally high-resolution images of the immediately adjacent tissues. To improve imaging of the pituitary gland, a receive-only surface coil that can be placed within the sphenoid sinus (the endosphenoidal coil [ESC]) during transsphenoidal surgery (TSS) was developed and assessed.

METHODS Five cadaver heads were used for preclinical testing of the ESC. The ESC (a double-turn, 12-mm-diameter surface coil made from 1-mm-diameter copper wire) was developed to obtain images in a 1.5-T MR scanner. The ESC was placed (via a standard sublabial TSS approach) on the anterior sella face. Clinical MR scans were obtained using the 8-channel head coil and ESC as the receiver coils. Using the ESC, ultra-high-resolution, 3D, balanced fast field echo (BFFE) and T1-weighted imaging were performed at resolutions of $0.25 \times 0.25 \times 0.50 \mathrm{~mm}^{3}$ and $0.15 \times 0.15 \times 0.30$ $\mathrm{mm}^{3}$, respectively.

RESULTS Region-of-interest analysis indicated a 10-fold increase in the signal-to-noise ratio (SNR) of the pituitary when using the ESC compared with the 8-channel head coil. ESC-related improvements $(p<0.01)$ in the SNR were inversely proportional to the distance from the ESC tip to the anterior pituitary gland surface. High-resolution BFFE MR imaging obtained using ESC revealed a number of anatomical features critical to pituitary surgery that were not visible on 8-channel MR imaging, including the pituitary capsule, the intercavernous sinus, and microcalcifications in the pars intermedia. These ESC imaging findings were confirmed by the pathological correlation with whole-mount pituitary sections.

CONCLUSIONS ESC can significantly improve SNR in the sellar region intraoperatively using current 1.5-T MR imaging platforms. Improvement in SNR can provide images of the sella and surrounding structures with unprecedented resolution. Clinical use of this ESC may allow for MR imaging detection of previously occult pituitary adenomas and identify microscopic invasion of the dura or cavernous sinus.

http://thejns.org/doi/abs/10.3171/2015.11.JNS151465

KEY WORDS endosphenoidal coil; transsphenoidal surgery; intraoperative magnetic resonance imaging; pituitary adenoma; pituitary surgery

\footnotetext{
ABBREVIATIONS BFFE = balanced fast field echo; $C D=$ Cushing's disease; $E S C=$ endosphenoidal coil; FFE = fast field echo; iMRI = intraoperative MRI; iUSG = intraoperative ultrasonography; $\mathrm{ROI}$ = region of interest; $\mathrm{SE}$ = spin echo; SNR = signal-to-noise ratio; T1-FFE = T1-weighted fast field echo; TSE = turbo spin echo; TSS = transsphenoidal surgery.

SUBMITTED June 23, 2015. ACCEPTED November 23, 2015.

INCLUDE WHEN CITING Published online March 18, 2016; DOI: 10.3171/2015.11.JNS151465.
} 
$\mathrm{S}$ UCCESSFUL treatment of pituitary adenomas is associated with the ability to detect the tumors and/or identify microscopic dural invasion on preoperative MR imaging. ${ }^{18,28}$ Currently, MR imaging with T1-weighted 2D spin-echo (SE) or 3D fast gradient echo sequences are used to detect pituitary microadenomas. ${ }^{24}$ Nevertheless, pituitary MR imaging (with and without intravenous contrast enhancement) for Cushing's disease (CD) fails to detect over $50 \%$ of adenomas and approximately $80 \%$ of microscopic dural invasion cases. ${ }^{18}$ When an adenoma and/or dural invasion is not identified on MR imaging before surgery, exploratory surgery is often not successful in curing the patient and, in many cases, eventually leads to radiation therapy and/or panhypopituitarism..$^{28}$ The inability to detect adenomas using MR imaging is due to small size and/or poor contrast-to-noise ratio. Furthermore, successful resection can be compromised by MR-invisible invasion of the sella dura, including the cavernous sinus wall(s). ${ }^{5}$

Currently, pituitary MR imaging is performed with MR receiver coils that are external to the head. The MR signal increases with the proximity of the receiver coil to the tissue being imaged. Consequently, it is advantageous to develop small coils that can be placed adjacent to the target tissue of interest and increase the signal-to-noise ratio (SNR) in the target organs. Specifically, coils that have been developed to provide high-resolution MR imaging include intravascular MR imaging coils for vascular angiography, ${ }_{1}^{15}$ intracavitary endoscope-mounted MR imaging coils for gastrointestinal imaging, ${ }^{21}$ and endorectal coils for MR imaging of the prostate. ${ }^{30}$ The increases in SNR can, for example, be used to increase resolution (when holding the imaging time constant) or image more rapidly (at the same resolution).

To improve imaging of the sella and detection of pituitary adenomas/dural invasion, we developed an endo- sphenoidal coil (ESC) for direct placement on the anterior sella face during transsphenoidal surgery (TSS) for intraoperative MR imaging. We hypothesized that the ESC would provide images with a higher SNR than that obtainable using standard phased-array coils and, consequently, could be used to generate higher resolution images of the sella and surrounding structures.

\section{Methods}

\section{Cadaveric Specimens}

Five human cadaveric heads were procured through the Maryland State Anatomy Board for imaging in an intraoperative MRI (iMRI) suite (Philips Achieva). The heads were preserved in alcohol-based preservative and injected with intravascular colored latex.

\section{ESC}

The ESC was designed to operate at 1.5 -T for ${ }^{1} \mathrm{H}$ MRI (64 MHz). The double-turn, 12-mm-diameter coil was made of 1.0-mm-diameter copper wire insulated with a Teflon hose $(0.1 \mathrm{~mm}$ thick). For safety, the coil was encased in 3-mm-thick plastic to ensure that a fixed minimum separation would be maintained between the tissue and the conductive elements of the coil when placed in the sphenoid sinus. The distal end of the coil casing was comprised of a silicone disc (1.0 mm thick) to visualize the coil position with respect to the pituitary using short TE gradient-echo MRI (Fig. 1). The coil was connected in parallel to a fixed capacitor and 14-cm-long, straight, twisted leads (1.0-mm-diameter copper wire) that extended the length of the nasal cavity (Fig. 1). The leads were insulated with Teflon hose (1 mm thick) and placed within a standard cable hose (10-mm outer diameter). The coil shaft containing the leads was semi-rigid and flexible.

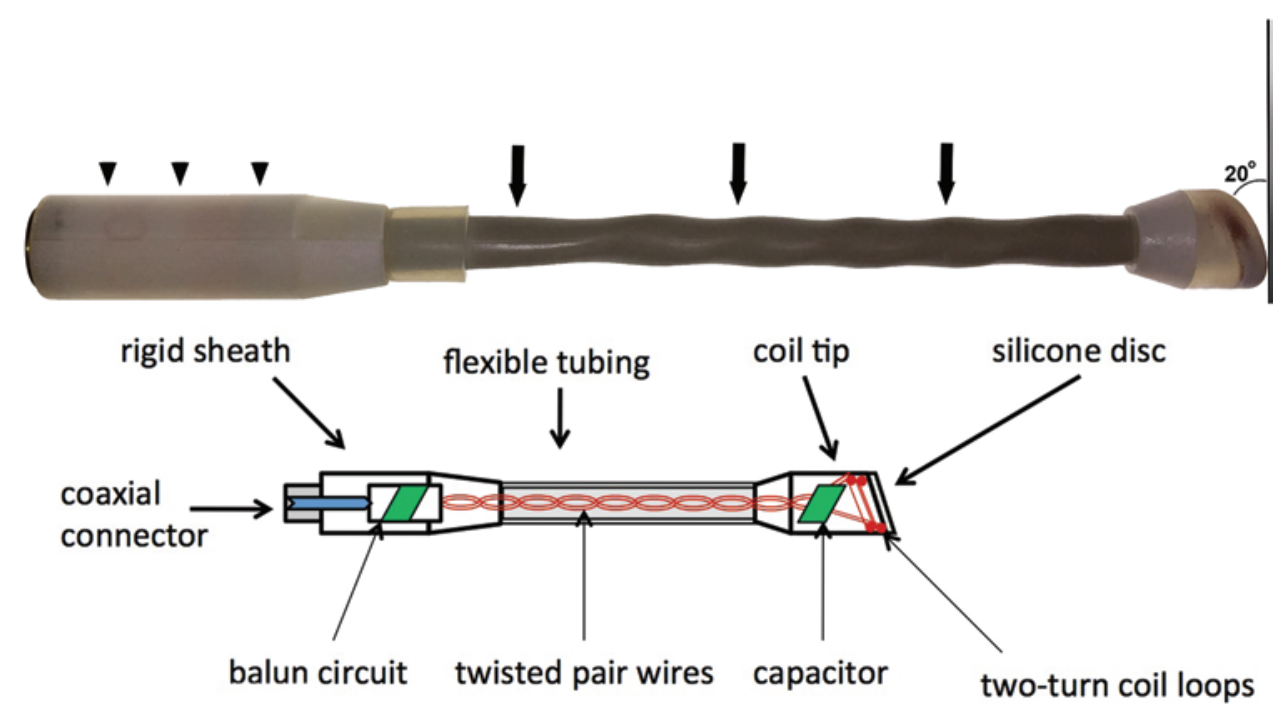

FIG. 1. Photograph (upper) and schematic diagram (lower) of the ESC. At the distal end, a beveled surface $\left(20^{\circ}\right.$ angle) comprised of a silicone disc separates the distal double turn of the coil from the tissue. The coil shaft is sheathed in a flexible tubing (arrows). Upper: A rigid sheath (arrowheads) provides a platform for securing the ESC to its holder. The entire coil housing is sealed and waterproof. Lower: The internal layout of the ESC consists of a distal coil within the coil tip, a semi-rigid twisted pair wires protected by flexible tubing, and the balun circuit protected by the proximal rigid sheath. Copyright Prashant Chittiboina. Published with permission. Figure is available in color online only. 
The coil plane was oriented at a $70^{\circ}$ angle with respect to the shaft to allow the coil to be placed as close as possible to the pituitary (Figs. 1 and 2). The coil leads were connected to a balun network and a coaxial cable connector within a rigid sheath (Fig. 1) that can be clamped into the coil holder and mounted onto the patient' bed (Fig. 2). The coil assembly was then connected to the manufacturer-provided interface box that was placed on the patient table using a cable protected with 4 braid wave traps. The interface box (Philips), which was originally designed to be used with endorectal coils, provided automatic tuning and matching of the coil and detuning of the coil during body coil radiofrequency transmission and signal preamplification. The cable traps were necessary to suppress the shield currents in the cables during radiofrequency transmission with the body coil. The interface box was in turn connected to the scanner receiver coil plug using a shielded cable.

\section{MR Imaging}

MR imaging was performed in an intraoperative MR suite using a 1.5-T MR scanner (Achieva, Philips Healthcare). A total of 9 MRI sessions (3 sessions for Specimen 1, 2 sessions for Specimen 2, 2 sessions for Specimen 3, and
1 session each for Specimens 4 and 5) were performed on the cadaver specimens. Initially, with the cadaver placed in the 8-channel coil, standard T1-weighted SE (FOV $18 \mathrm{~cm}$; image resolution $0.625 \times 0.625 \times 3 \mathrm{~mm}$; TR/TE 400/10.5 msec; scan time 1 minute and 55 seconds), and T2-weighted turbo SE (TSE) (FOV $12 \mathrm{~cm}$; image resolution $0.5 \times 0.5 \times 3 \mathrm{~mm}$; TR/TE $3000 / 100 \mathrm{msec}$, scan time 6 minutes and 24 seconds) of the cadaver's pituitary were obtained. The 8-channel array was then removed, and the ESC was inserted into the sphenoid sinus of the cadaver through the operative corridor (Fig. 2). T1-weighted SE and T2-weighted TSE images were then repeated using the ESC as the receiver coil. In addition, high-resolution, T2-weighted, TSE images were acquired with a reduced FOV of $4 \mathrm{~cm}$ and resolution of $0.5 \times 0.5 \times 1.5 \mathrm{~mm}$ in 2 minutes and 24 seconds. Ultra-high-resolution 3D fast field echo (FFE) sequences were obtained using balanced fast field echo (BFFE) and T1-weighted (T1-FFE) techniques. BFFE images (FOV $6 \mathrm{~cm}$; image resolution $0.25 \times$ $0.25 \times 0.50 \mathrm{~mm}$; TR/TE $9.3 / 3.3 \mathrm{msec}$; flip angle $25^{\circ}$; scan time 6 minutes and 21 seconds) were obtained twice with different phase offsets and combined to remove banding artifacts. T1-FFE images (FOV $6 \mathrm{~cm}$; image resolution $0.15 \times 0.15 \times 0.3 \mathrm{~mm}$; TR/TE $15.5 / 5.3 \mathrm{~ms}$; flip angle $10^{\circ}$;
A

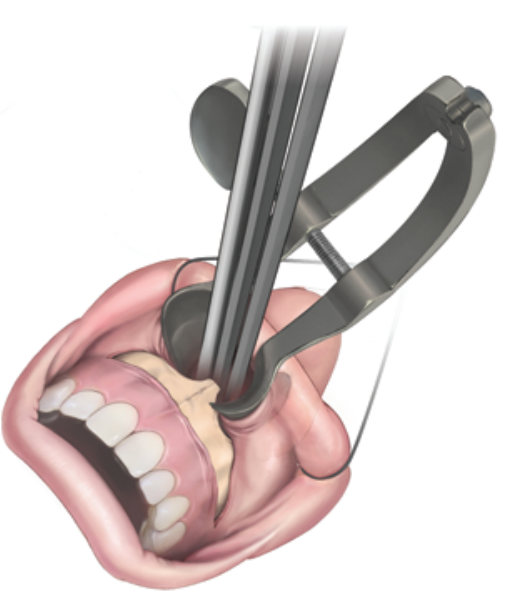

B

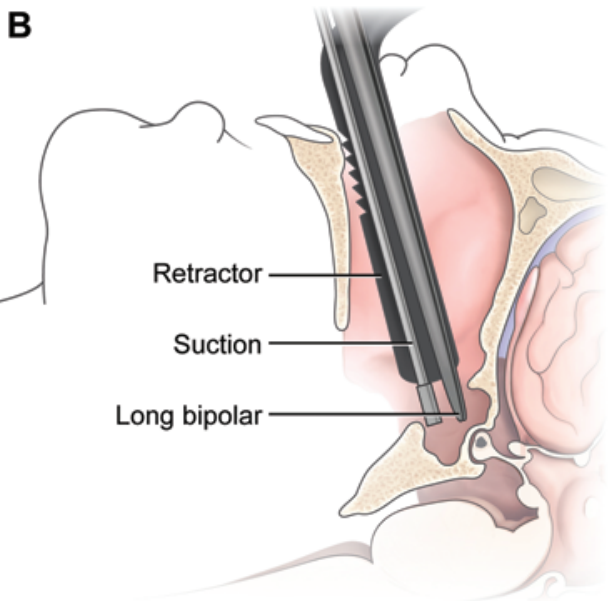

C
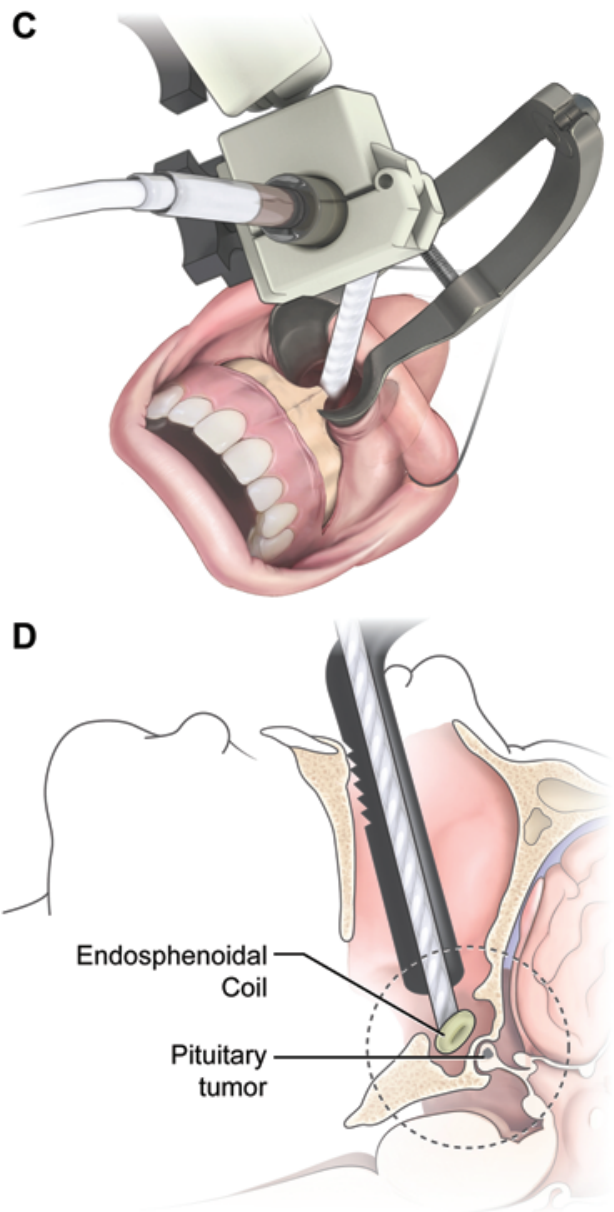

FIG. 2. A and B: The sublabial transsphenoidal approach creates a surgical corridor for the instruments and ESC. C and D: The ESC is designed to be inserted through the surgical corridor in order to reach the sphenoid sinus. The dotted circle in panel D estimates the region that is imaged with ESC. Copyright Prashant Chittiboina. Published with permission. 
scan time 20 minutes and 20 seconds) were also acquired with the ESC.

\section{Coil Placement}

A standard sublabial TSS approach was performed in all cadaver heads to allow for the placement of the ESC through the operative corridor. ${ }^{20}$ The cadaver head was fixed in a 3-pin, MR-compatible fixation device. ${ }^{37}$ The bone overlying the anterior face and floor of the sella was left intact. The ESC was sheathed in a waterproof latex or polyethylene sleeve and placed within the surgical corridor under direct vision (Fig. 2). The beveled leading edge of the coil was positioned immediately adjacent to the anterior aspect of anterior pituitary gland. Once a satisfactory position was achieved under direct vision, the coil was fixed in position with a custom-designed holder with 6 degrees of freedom. The base of the coil holder was firmly attached to the head fixation device. The cadaver head and coil were then moved to the scanner isocenter.

\section{Imaging Analysis}

The SNR was measured on T1-weighted SE images that were obtained using ESC in each of the 9 studies in a standard manner. Briefly, the signals within a region of interest (ROI) placed in the pituitary gland (the target tissue of interest) and in the air of the sphenoid sinus (any signal present in the air should be noise) were measured. The SNR of each image was calculated as the mean of the signal ROI placed within the pituitary divided by the standard deviation of the noise ROI. The standard deviation of the signal measured within the air is an accepted measure of the noise of an MR acquisition. ${ }^{11}$ SNR measurements from different receiver coils were compared using the Student t-test.

\section{Histopathology}

Histopathological analysis was performed on wholemount pituitary gland sections after imaging a representative cadaver head. The pituitary gland and parasellar structures were carefully dissected and removed en bloc. Decalcification of the specimen was performed with diluted decalcifying solution (Surgipath Decalcifier II; Leica Biosystems Nussloch, $\mathrm{GmbH}$ ) for 72 hours. Following this, 2- to 3-mm-thick coronal sections were made and embedded in paraffin blocks. Standard histopathological sections (5 $\mu \mathrm{m}$ thick) were stained with $\mathrm{H} \& \mathrm{E}$ and Masson's trichrome.

\section{Results \\ ESC Placement}

Placement of the ESC through the standard sublabial TSS was successfully performed on all 5 cadaveric specimens. The ESC was easily accommodated and placed to the target depth anterior to the anterior sella dura through the standard TSS surgical corridor in all cases. The initial MR images following ESC insertion were used to measure the distance between the ESC tip and the surface of the pituitary gland. The distance ranged from 4.2 to $17 \mathrm{~mm}$.

\section{Pituitary Imaging \\ SNR}

Using either the 8-channel coil or ESC, the pituitary gland and larger anatomical surrounding structures could be clearly defined. Marked improvement in SNR within the pituitary was obtained with ESC (Fig. 3), as measured on T1-weighted SE images. SNR varied inversely with the distance of the ESC tip to the surface of the pituitary gland. When the ESC tip was positioned closer (less than $6 \mathrm{~mm}$ ) to the pituitary surface (4 studies), SNR was 5 to 12 (mean $10.9 \pm 1.6$ ) times that obtained using the standard 8 -channel coil $(\mathrm{p}<0.01)$. When the ESC tip was placed farther $(9-17 \mathrm{~mm}$ ) from the pituitary surface (5 studies), SNR was 3 to 5 (mean $4.2 \pm 0.7$ ) times that obtained using the standard 8 -channel coil $(\mathrm{p}<0.01)$. As expected, a higher ESC SNR was associated with a steeper drop-off in distance compared with the standard 8-channel head

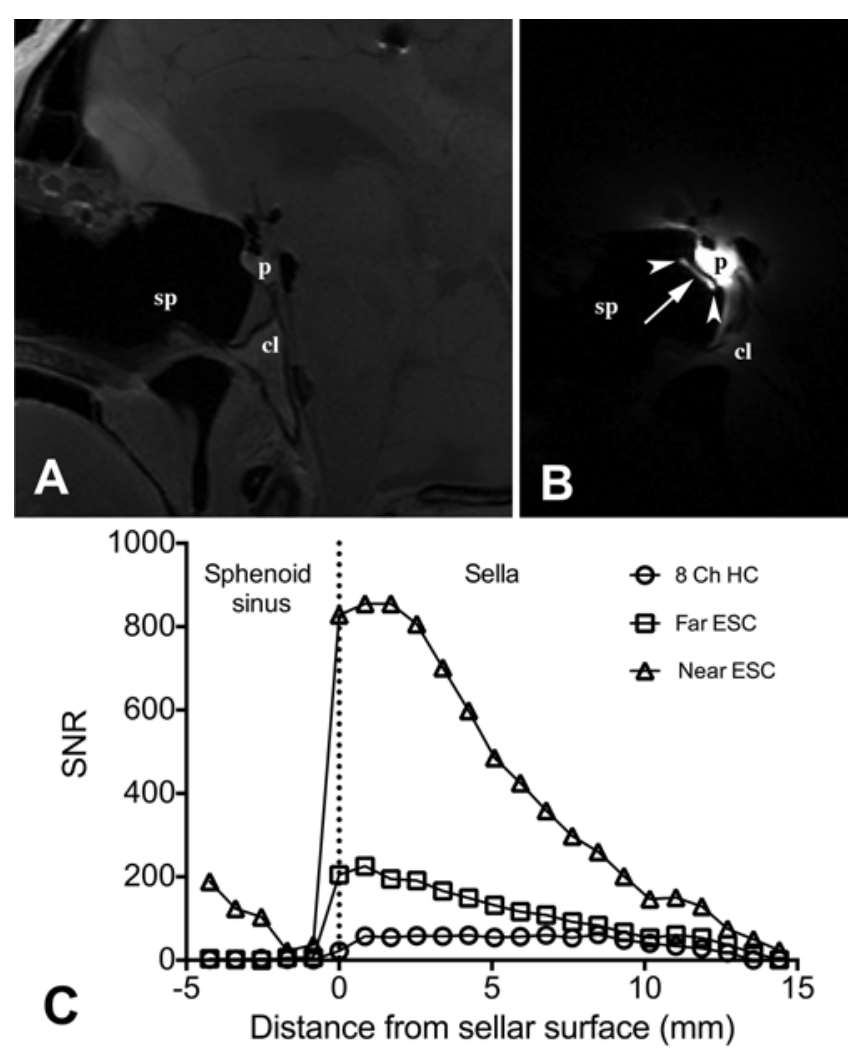

FIG. 3. A and B: Comparative T1-weighted SE images (resolution 0.625 $\left.\times 0.625 \times 3 \mathrm{~mm}^{3}\right)$ of the pituitary gland of a cadaver head obtained using the 8-channel coil (A) and ESC (B). Images are shown with the same window and level display settings. Markedly improved SNR within the pituitary is obtained with the ESC. The white arrowheads indicate the location of the silicone discs at the distal ESC. The distance between the center of the ESC (white arrow) and the pituitary surface is $3.2 \mathrm{~mm}$. In these images, the SNR values within the pituitary gland when using the 8-channel coil and the ESC are 73 and 780, respectively. C: Comparison of the SNR line profiles through the pituitary for the 8-channel head coil and 2 different ESC placements. When placement was $4 \mathrm{~mm}$ from the pituitary surface (near the ESC), the SNR gains were much greater than when the ESC was placed $\sim 10 \mathrm{~mm}$ from the pituitary surface (far from the ESC). A steep drop-off in SNR with increasing distance is evident using ESC when compared with the 8-channel head coil. $8 \mathrm{Ch} \mathrm{HC}=8$ channel head coil; $c l=$ clivus; $p=$ pituitary gland; $s p=$ sphenoid sinus. 
coil. When the ESC was positioned closer to the sella, the peak SNR with the ESC was 14.8-fold higher than that of the standard 8-channel coil (SNR 854 and 57, respectively). Peak SNR dropped dramatically (SNR 225) when the ESC was positioned further from the pituitary gland (Fig. 3).

\section{Image Acquisition Time}

The coronal T2-weighted images obtained with the standard 8-channel head coil using an FOV of $120 \mathrm{~mm}$ and a matrix size of 240 were compared with those obtained using the ESC and an identical acquisition matrix. Using the ESC with an FOV that was reduced by a factor of 3 resulted in a corresponding decrease in acquisition time. Visually, there is no degradation of image quality on the faster sequence (Fig. 4).

\section{Image Resolution}

The improved SNR afforded by ESC allowed the acquisition of good-quality images at resolutions not feasible with the standard 8-channel head coil. A number of anatomical details emerged on the $3 \mathrm{D} \operatorname{BFFE}(0.25 \times 0.25 \times$ $\left.0.50 \mathrm{~mm}^{3}\right)$ and 3D T1-FFE $\left(0.15 \times 0.15 \times 0.3 \mathrm{~mm}^{3}\right)$ images (Fig. 5) that were not visible with the 8-channel head coil, including the pituitary capsule, intercavernous (coronal) sinus, and pars intermedia microcalcification. Histological evaluation of the same cadaver confirmed these structures to be present histologically (Fig. 6).

\section{Discussion}

\section{Current Methods for Enhancing Imaging of the Sella}

Because of the limitations of preoperative MR imaging of the sella, surgeons have used intraoperative methods in an attempt to provide clinically important information. Both intraoperative ultrasonography (iUSG) and iMRI have been described for surgery of the pituitary gland. Previously, iUSG was performed by inserting an ultrasonography probe through the TSS surgical corridor. The use of iUSG has permitted the detection of tumor residuals and the identification of large critical structures, including the carotid arteries..$^{2,23,32}$ However, iUSG failed to reliably detect MR-invisible microadenomas in CD patients due to limited resolution, limited directionality, small FOV size, and artifacts. ${ }^{8}$ Recently, iMRI in association with TSS has been reported. ${ }^{4,5,33}$ These reports used receiver coils that are external to the head, so the SNR of such methods can
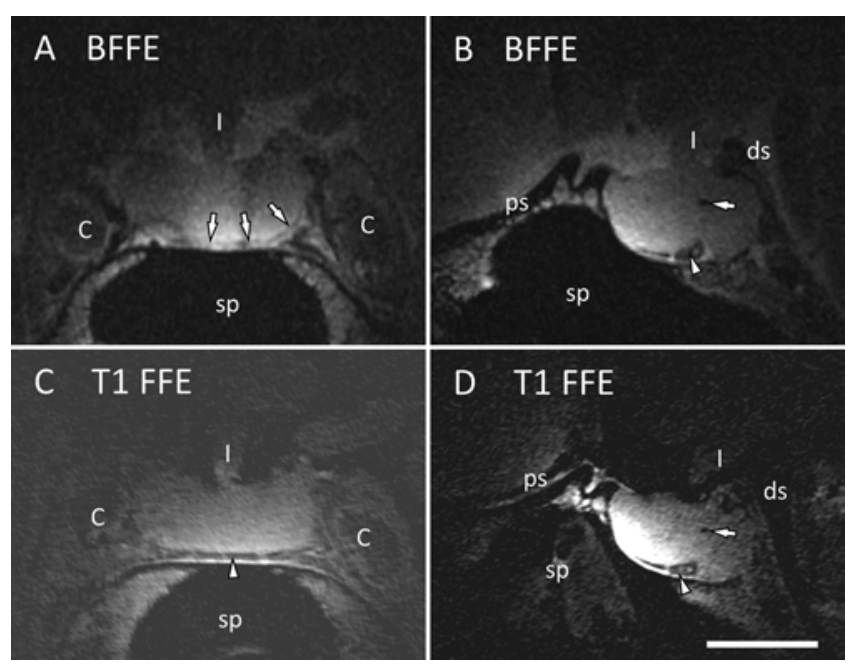

FIG. 5. Ultra-high-resolution images of the cadaver pituitary gland obtained using the ESC reformatted in the coronal ( $A$ and $C$ ) and sagittal $(B$ and $D)$ planes. A microcalcification is seen on the both sequences ( $B$ and $D$, arrow). The pituitary capsule is identified on the coronal BFFE image ( $A$, arrows). The inferior intercavernous sinus is identified on both sequences ( $A, C$, and $D$, arrowheads). Scale bar $=1 \mathrm{~cm}$. 3D BFFE is $0.25 \times 0.25 \times 0.50 \mathrm{~mm}^{3}$ and $3 \mathrm{D} \mathrm{T1}$-FFE is $0.15 \times 0.15 \times 0.3 \mathrm{~mm}^{3} . \mathrm{C}=$ internal carotid artery; $d s=$ dorsum sellae; $\mathrm{I}=$ infundibulum; $\mathrm{ps}=$ planum sphenoidale; $s p=$ sphenoid sinus.

be no better than that performed preoperatively. Nevertheless, the advantages of iMRI with a standard head could include the detection of larger tumor remnants and cerebrospinal fluid leakage. ${ }^{12}$

\section{Current Study}

Feasibility of ESC

ESC was specifically designed as an adjunct iMRI tool to improve imaging of the sella and parasellar structures. The ESC includes a number of mechanical and electrical features that facilitate placement and ensure safety for use during TSS. The distal double/triple-turn coil is at an acute angle $\left(70^{\circ}\right)$ to the axis of the coil (Fig. 2). This makes the distal surface beveled and optimally angled for placement close to the anterior face of the sella (Figs. 1-3). The coil is housed within a water-tight enclosure that provides electrical and thermal insulation. The inclusion of a silicone disc at the distal end allows for the detection of the distal end of the coil during MR imaging, thus allowing adjust-
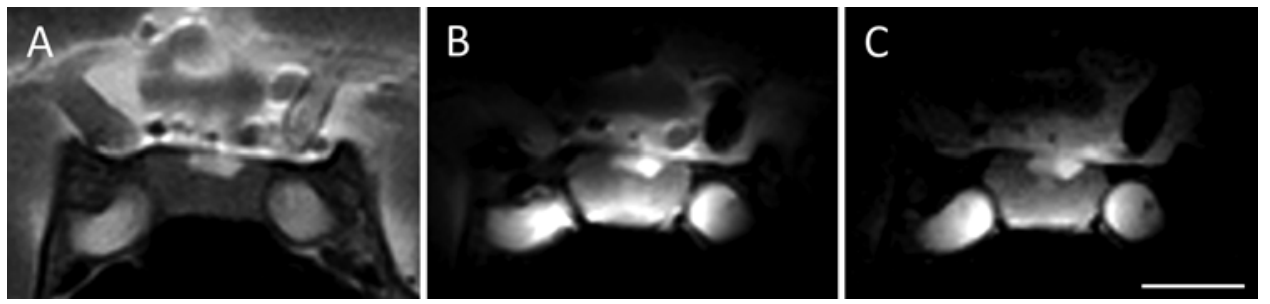

FIG. 4. Comparison of clinical T2-weighted TSE coronal images obtained with an 8-channel head coil (A) and ESC using the same (B) and decreased (C) scan times. Using a $\sim 6$ minute and 30 second acquisition time at a resolution of $0.5 \times 0.5 \times 3 \mathrm{~mm}$, images from both coils ( $A$ and $B$ ) contained similar anatomical detail, but higher SNR is shown using ESC $(B)$. The 3-fold reduction in scan time (2 minutes and 24 seconds) results in no visible degradation of image quality $(C)$ with the same in-plane resolution and half the slice thickness $(0.5 \times 0.5 \times 1.5 \mathrm{~mm})$. Scale bar $=1 \mathrm{~cm}$. 


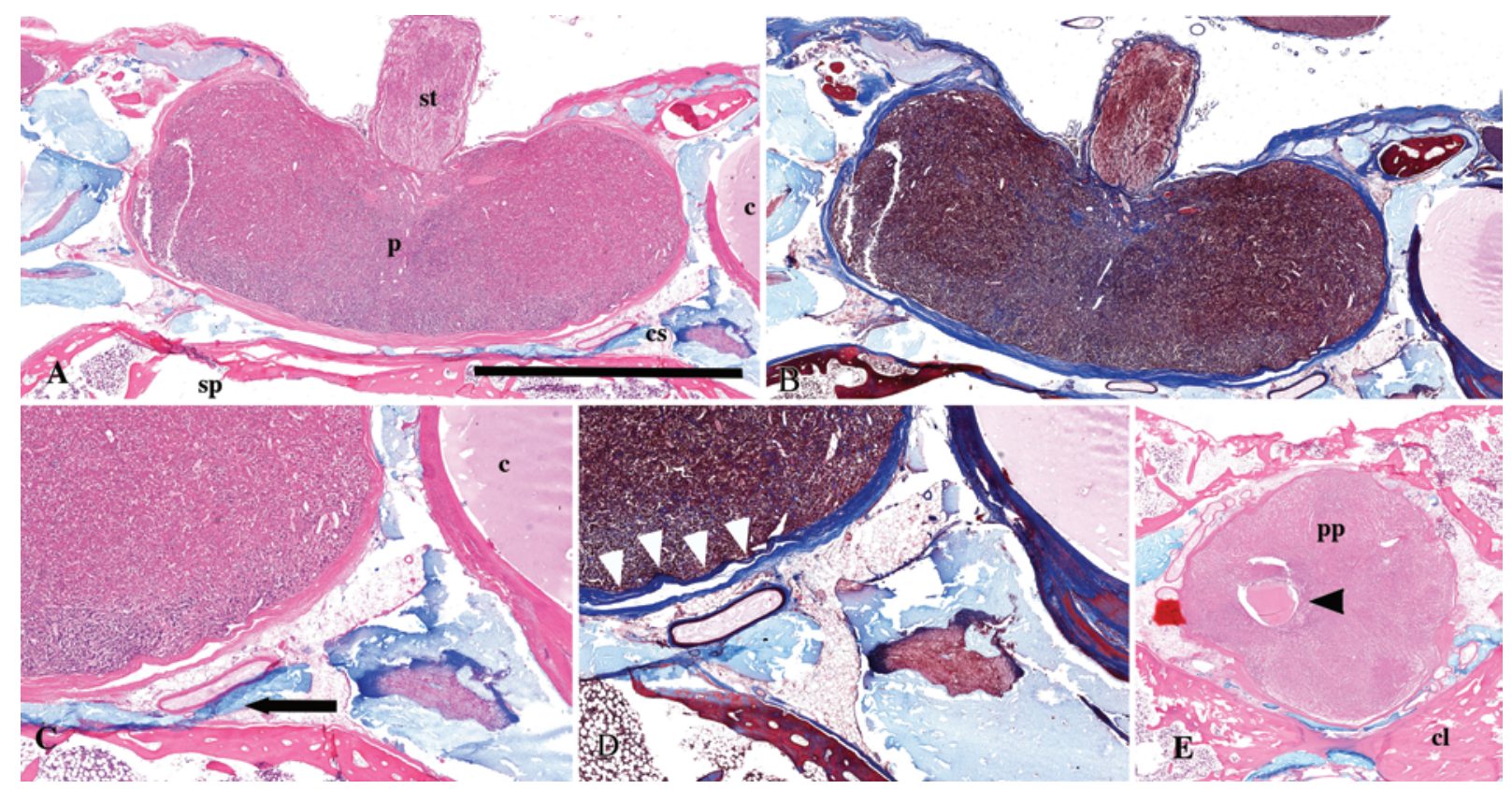

FIG. 6. Whole-mount pituitary gland specimen from a cadaver were examined for confirmation of the anatomical features (the specimen corresponding to Fig. 5 is shown here). After decalcification, H \& E staining (A, C, and E) and Masson's trichrome staining ( $B$ and $\mathbf{D})$ was performed. $C$ : The injected blue latex channel, corresponding to the intercavernous sinus, was seen inferior to the gland (black arrow). D: The pituitary capsule was well visualized as an adherent connective tissue layer apposed to the gland surface (white arrowheads) and separate from the adjacent dura and cavernous sinus wall. E: Microcalcification in the posterior pituitary gland (pp) was also confirmed on the $\mathrm{H} \& \mathrm{E}$ section through the posterior pituitary gland (black arrowhead). Scale bar $=1$ $\mathrm{cm} . \mathrm{c}=$ cavernous segment of internal carotid artery; $\mathrm{cl}=$ clivus; $\mathrm{p}=$ pituitary gland; $\mathrm{sp}=$ sphenoid sinus; st = pituitary stalk.

ment of the coil position. The shaft of ESC is semi-rigid in order to allow deformation, bending, and facilitate the optimal placement of the coil within the surgical corridor. In this feasibility study, we demonstrate that the placement of the ESC through the transsphenoidal route can be performed, allowing high-resolution imaging of the pituitary gland and surrounding structures.

\section{ESC Improves SNR}

In MR imaging, increased SNR improves the contrastto-noise ratio between the tissues of interest. Alternatively, the increase in SNR can be traded to generate either improved spatial resolution or decreased imaging time. Improvements in SNR are achieved using a higher magnetic field strength (which leads to a linear improvement in SNR) $)^{19,29}$ and receiver coils that can be placed close to anatomical sites of interest. Such coils were first described to detect chemical compounds in localized regions adjacent to the coils. ${ }^{1}$ The disadvantage of such coils is that the signal is mainly detected from the tissue within a radius that is comparable to that of the coil itself, resulting in a limited field of view. This limitation can be partially overcome by the use of phased-array coils (multiple overlapping surface coils that operate independently), in which the combined signal results in a composite image with a higher SNR that is close to the individual coil elements and a larger FOV. However, there is limited or no improvement in SNR for tissues that are far from all of the individual coil elements.

Currently, preoperative MR imaging of the pituitary gland most commonly employs external phased-array head coils or, in intraoperative cases, external surface coils. The pituitary gland, which is situated at the center of the head, is a poor target for externally placed coils because of its distance from the individual coil elements. We have shown that the ESC described in this work, when placed close to the pituitary gland, results in an approximately 10 -fold increase in the SNR compared with the standard 8-channel head coil (Fig. 3). Such an increase in SNR by increasing the field strength alone is not feasible with current MR technology. By using ESC, we have also shown that the improvement in SNR can be used to generate ultra-highresolution images of the sella (Fig. 5). Alternatively, faster imaging can be performed (Fig. 4).

\section{Potential Applications}

\section{Detection of Microadenomas}

An increase in the MR imaging field strength from 1.5 $\mathrm{T}$ to $3 \mathrm{~T}$ results in a modest improvement in the sensitivity for detecting pituitary microadenoma..$^{14,26,27}$ The improvement in sensitivity is due to a modest increase in SNR and consequent improvement in the image resolution. In the current study, we were able to generate ultra-high-resolution images of the pituitary gland without image degradation (Figs. 4 and 5). Such images allowed for the detection of very small anatomical features, including the pituitary capsule and microcalcifications within the pituitary gland. We anticipate that, when used in clinical settings with intravenous contrast infusion, such high-resolution images have the potential to dramatically improve the sensitivity of the detection of microadenomas. 
Currently, dynamic pituitary imaging is limited to sections that are at least $2 \mathrm{~mm}$ thick across the sella and an imaging time of 2 to 4 minutes. ${ }^{13,16}$ However, differential enhancement within the pituitary gland may best be visible during the first 10 to 20 seconds..$^{10}$ The ESC can potentially allow the fast acquisition of higher resolution sellar images. Obtaining higher resolution images during dynamic imaging may improve the detection of smaller adenomas by preventing the tissue averaging seen in thicker slices. ${ }^{13,24}$

\section{Detection of Cavernous Sinus Invasion}

Using current MR imaging techniques, cavernous sinus invasion by an adenoma is accurately detected in only $22 \%$ of the cases. ${ }^{17}$ Microscopic dural invasion (without gross cavernous sinus invasion) remains undetected in $100 \%$ of patients. ${ }^{18}$ Undetected microscopic dural invasion is associated with treatment failure in CD patients and patients with other pituitary tumors. ${ }^{7,22}$ Preoperatively, surgeons depend on indirect predictors of cavernous sinus invasion that do not provide detailed information about dural invasion and the size of defects in the medial cavernous sinus wall. ${ }^{36}$ Modest improvements in SNR (approximately 2-fold) with 3.0-T MR imaging has been shown to improve the detection of cavernous sinus invasion. ${ }^{6}$ We have found that ESC resulted in an approximately 10 -fold improvement in SNR in the sella/pituitary gland.

This study demonstrates that normal parasellar structures, including the pituitary capsule and medial cavernous sinus walls, are visualized with a high resolution (Figs. 5 and 6). Such high-resolution imaging may help with the intraoperative detection of small or microscopic foci of dural invasion, allowing for immediate resection of the involved dura or medial cavernous sinus wall. In CD patients and patients with other pituitary tumors, gross and microscopic dural invasion leads to treatment failure.,22 More accurate imaging of the cavernous sinus and other sellar dural invasions can lead to a targeted surgical approach and improve long-term outcomes., ${ }^{9,35}$ Additionally, intraoperative detection of gross cavernous sinus invasion may lead to earlier postoperative radiation of tumor remnants, leading to improved long-term remission rates.

In the current implementation, the placement of the ESC for imaging requires a surgical corridor, and therefore the initial application of this device will be confined to intraoperative settings. The initial indications for use that motivated the development of the coil is the localization of MRI-invisible microadenomas in patients with CD and strong biochemical evidence for sellar adrenocorticotropic hormone-producing adenoma. Typically, such evidence includes inferior petrosal sinus sampling data. ${ }^{3}$ In this context, surgeons perform exploration of the pituitary gland and partial hypophysectomy if an adenoma is not found intraoperatively. ${ }^{18,31}$ It is hoped that the intraoperative placement of the ESC will allow the detection of the previously invisible adenoma, leading to a directed approach to selective adenectomy. Furthermore, we anticipate that the ESC can be advantageous even in cases in which adenomas are clearly visible on preoperative MR images. The ESC may allow the detection of previously invisible sites of adenoma invasion ${ }^{17}$ and allow real-time modifications in surgical planning to resect the regions of the involved medial wall of the cavernous sinus or dura. The detection of the microscopic sites of adenoma invasion has the potential to improve long-term outcomes in CD patients. ${ }^{7,22}$

\section{Limitations}

The current study was performed in cadavers; therefore, several caveats are recognized for the application of ESC in clinical settings. The feasibility of the placement of ESC was tested in cadavers, whose water content, tissue tensile strength, and deformation differ significantly from human patients. The loss of capillary perfusion and tissue water motility may lead to inadequate imaging of tissue compartmentalization. ${ }^{25}$ This may also lead to the inadequate detection of real pathology that may have existed in the cadaver specimens. It is also not clear if anatomical variations in sphenoid and sellar morphology will prevent the optimal placement of the ESC. The limited FOV and contamination with air and blood may limit the benefits of ESC when evaluating residual tumors during the resection of large macroadenomas. The distal residuals of the lesion may be beyond the range of the ESC. Such a limitation may be overcome by developing coil configurations that allow the simultaneous use of ESC and external coils.

The current ESC assembly was designed to meet the requirements for placement via the sublabial transsphenoidal approach. As such, further modification of the coil would be required for use via endonasal or endoscopic approaches. ${ }^{34}$ The most significant limitation of the study is that we have not directly evaluated cadavers (or living patients) with microadenomas. Because contrast injection is necessary to detect microadenomas, the definitive assessment of ESC for the detection of (currently) MR imaging-occult microadenomas awaits trial in a patient population.

\section{Conclusions}

This cadaveric study demonstrates that using ESC and available iMRI platforms is a valid strategy for improving SNR by approximately 10 -fold in the sellar region intraoperatively. Increased SNR should lead to the improved detection of small microadenomas during TSS and far superior visualization of the sellar structures, including the medial wall of the cavernous sinus. Consequently, previously undetectable adenomas and subtle microscopic tumor invasion may now be detectable, leading to the improved surgical management of pituitary tumors.

\section{Acknowledgments}

This study was funded by the intramural research program of the National Institute of Neurological Disorders and Stroke at the National Institutes of Health (NIH). This research was also made possible through the NIH Medical Research Scholars Program, a public-private partnership supported jointly by the NIH and generous contributions to the Foundation for the NIH.

\section{References}

1. Ackerman JJ, Grove TH, Wong GG, Gadian DG, Radda GK: Mapping of metabolites in whole animals by ${ }^{31} \mathrm{P}$ NMR using surface coils. Nature 283:167-170, 1980 
2. Arita K, Kurisu K, Tominaga A, Kawamoto H, Iida K, Mizoue T, et al: Trans-sellar color Doppler ultrasonography during transsphenoidal surgery. Neurosurgery 42:81-86, 1998

3. Arnaldi G, Angeli A, Atkinson AB, Bertagna X, Cavagnini F, Chrousos GP, et al: Diagnosis and complications of Cushing's syndrome: a consensus statement. J Clin Endocrinol Metab 88:5593-5602, 2003

4. Black PM, Moriarty T, Alexander E III, Stieg P, Woodard EJ, Gleason PL, et al: Development and implementation of intraoperative magnetic resonance imaging and its neurosurgical applications. Neurosurgery 41:831-845, 1997

5. Buchfelder M, Schlaffer SM: Intraoperative magnetic resonance imaging during surgery for pituitary adenomas: pros and cons. Endocrine 42:483-495, 2012

6. Cao L, Chen H, Hong J, Ma M, Zhong Q, Wang S: Magnetic resonance imaging appearance of the medial wall of the cavernous sinus for the assessment of cavernous sinus invasion by pituitary adenomas. J Neuroradiol 40:245-251, 2013

7. Dickerman RD, Oldfield EH: Basis of persistent and recurrent Cushing disease: an analysis of findings at repeated pituitary surgery. J Neurosurg 97:1343-1349, 2002

8. Doppman JL, Ram Z, Shawker TH, Oldfield EH: Intraoperative US of the pituitary gland. Work in progress. Radiology 192:111-115, 1994

9. Fahlbusch R, von Keller B, Ganslandt O, Kreutzer J, Nimsky $\mathrm{C}$ : Transsphenoidal surgery in acromegaly investigated by intraoperative high-field magnetic resonance imaging. Eur J Endocrinol 153:239-248, 2005

10. Finelli DA, Kaufman B: Varied microcirculation of pituitary adenomas at rapid, dynamic, contrast-enhanced MR imaging. Radiology 189:205-210, 1993

11. Firbank MJ, Coulthard A, Harrison RM, Williams ED: A comparison of two methods for measuring the signal to noise ratio on MR images. Phys Med Biol 44:N261-N264, 1999

12. Ginat DT, Swearingen B, Curry W, Cahill D, Madsen J, Schaefer PW: 3 Tesla intraoperative MRI for brain tumor surgery. J Magn Reson Imaging 39:1357-1365, 2014

13. Kasaliwal R, Sankhe SS, Lila AR, Budyal SR, Jagtap VS, Sarathi V, et al: Volume interpolated 3D-spoiled gradient echo sequence is better than dynamic contrast spin echo sequence for MRI detection of corticotropin secreting pituitary microadenomas. Clin Endocrinol (Oxf) 78:825830, 2013

14. Kim LJ, Lekovic GP, White WL, Karis J: Preliminary experience with 3-Tesla MRI and Cushing's disease. Skull Base 17:273-277, 2007

15. Lederman RJ: Cardiovascular interventional magnetic resonance imaging. Circulation 112:3009-3017, 2005

16. Lee HB, Kim ST, Kim HJ, Kim KH, Jeon P, Byun HS, et al: Usefulness of the dynamic gadolinium-enhanced magnetic resonance imaging with simultaneous acquisition of coronal and sagittal planes for detection of pituitary microadenomas. Eur Radiol 22:514-518, 2012

17. Lonser RR, Ksendzovsky A, Wind JJ, Vortmeyer AO, Oldfield EH: Prospective evaluation of the characteristics and incidence of adenoma-associated dural invasion in Cushing disease. J Neurosurg 116:272-279, 2012

18. Lonser RR, Wind JJ, Nieman LK, Weil RJ, DeVroom HL, Oldfield EH: Outcome of surgical treatment of 200 children with Cushing's disease. J Clin Endocrinol Metab 98:892901, 2013

19. Magee T, Shapiro M, Williams D: Comparison of high-fieldstrength versus low-field-strength MRI of the shoulder. AJR Am J Roentgenol 181:1211-1215, 2003

20. Mason RB, Nieman LK, Doppman JL, Oldfield EH: Selective excision of adenomas originating in or extending into the pituitary stalk with preservation of pituitary function. $\mathbf{J}$ Neurosurg 87:343-351, 1997

21. Matsuoka Y, Takahashi A, Kumamoto E, Morita Y, Kutsumi H, Azuma T, et al: High-resolution MR imaging of gastrointestinal tissue by intracavitary RF coil with remote tuning and matching technique for integrated MR-endoscope system. Conf Proc IEEE Eng Med Biol Soc 2013:57065710,2013

22. Meij BP, Lopes MBS, Ellegala DB, Alden TD, Laws ER Jr: The long-term significance of microscopic dural invasion in 354 patients with pituitary adenomas treated with transsphenoidal surgery. J Neurosurg 96:195-208, 2002

23. Ota Y, Mami I: Ultrasonography imaging during nasal endoscopic transsphenoidal surgery. ORL J Otorhinolaryngol Relat Spec 75:27-31, 2013

24. Patronas N, Bulakbasi N, Stratakis CA, Lafferty A, Oldfield EH, Doppman J, et al: Spoiled gradient recalled acquisition in the steady state technique is superior to conventional postcontrast spin echo technique for magnetic resonance imaging detection of adrenocorticotropin-secreting pituitary tumors. J Clin Endocrinol Metab 88:1565-1569, 2003

25. Pfefferbaum A, Sullivan EV, Adalsteinsson E, Garrick T, Harper C: Postmortem MR imaging of formalin-fixed human brain. Neuroimage 21:1585-1595, 2004

26. Pinker K, Ba-Ssalamah A, Wolfsberger S, Mlynarik V, Knosp E, Trattnig S: The value of high-field MRI (3T) in the assessment of sellar lesions. Eur J Radiol 54:327-334, 2005

27. Portocarrero-Ortiz L, Bonifacio-Delgadillo D, SotomayorGonzález A, Garcia-Marquez A, Lopez-Serna R: A modified protocol using half-dose gadolinium in dynamic 3-Tesla magnetic resonance imaging for detection of ACTHsecreting pituitary tumors. Pituitary 13:230-235, 2010

28. Pouratian N, Prevedello DM, Jagannathan J, Lopes MB, Vance ML, Laws ER Jr: Outcomes and management of patients with Cushing's disease without pathological confirmation of tumor resection after transsphenoidal surgery. J Clin Endocrinol Metab 92:3383-3388, 2007

29. Redpath TW: Signal-to-noise ratio in MRI. Br J Radiol 71:704-707, 1998

30. Schnall MD, Lenkinski RE, Pollack HM, Imai Y, Kressel HY: Prostate: MR imaging with an endorectal surface coil. Radiology 172:570-574, 1989

31. Semple PL, Vance ML, Findling J, Laws ER Jr: Transsphenoidal surgery for Cushing's disease: outcome in patients with a normal magnetic resonance imaging scan. Neurosurgery 46:553-559, 2000

32. Solheim O, Selbekk T, Løvstakken L, Tangen GA, Solberg OV, Johansen TF, et al: Intrasellar ultrasound in transsphenoidal surgery: a novel technique. Neurosurgery 66:173-186, 2010

33. Steinmeier R, Fahlbusch R, Ganslandt O, Nimsky C, Buchfelder M, Kaus M, et al: Intraoperative magnetic resonance imaging with the Magnetom Open scanner: concepts, neurosurgical indications, and procedures: a preliminary report. Neurosurgery 43:739-748, 1998

34. Swearingen B: Update on pituitary surgery. J Clin Endocrinol Metab 97:1073-1081, 2012

35. Tanei T, Nagatani T, Nakahara N, Watanabe T, Nishihata T, Nielsen ML, et al: Use of high-field intraoperative magnetic resonance imaging during endoscopic transsphenoidal surgery for functioning pituitary microadenomas and small adenomas located in the intrasellar region. Neurol Med Chir (Tokyo) 53:501-510, 2013

36. Vieira JO Jr, Cukiert A, Liberman B: Magnetic resonance imaging of cavernous sinus invasion by pituitary adenoma diagnostic criteria and surgical findings. Arq Neuropsiquiatr 62 (2B):437-443, 2004

37. Weingarten DM, Asthagiri AR, Butman JA, Sato S, Wiggs EA, Damaska B, et al: Cortical mapping and frameless 
stereotactic navigation in the high-field intraoperative magnetic resonance imaging suite. J Neurosurg 111:11851190,2009

\section{Disclosures}

The authors report no conflict of interest concerning the materials or methods used in this study or the findings specified in this paper.

\section{Author Contributions}

Conception and design: Chittiboina, Talagala, Merkle, Lonser, Oldfield, Koretsky, Butman. Acquisition of data: Chittiboina, Talagala, Merkle, Sarlls, Montgomery, Piazza, Ray-Chaudhury,
Butman. Analysis and interpretation of data: Chittiboina, Talagala, Sarlls, Butman. Drafting the article: Chittiboina. Critically revising the article: Chittiboina, Talagala, Lonser, Oldfield, Koretsky, Butman. Reviewed submitted version of manuscript: Chittiboina, Talagala, Lonser, Butman. Approved the final version of the manuscript on behalf of all authors: Chittiboina. Administrative/technical/material support: Montgomery, Piazza, Scott. Study supervision: Koretsky, Butman.

\section{Correspondence}

Prashant Chittiboina, Surgical Neurology Branch, National Institute of Neurological Disorders and Stroke, National Institutes of Health, 10 Center Dr., Rm. 3D20, Bethesda, MD 20892-1414. email: prashant.chittiboina@nih.gov. 